\title{
VISUAL AND STATISTICAL ANALYSIS OF DIGITAL ELEVATION MODELS GENERATED USING IDW INTERPOLATOR WITH VARYING POWERS
}

\author{
F. F. Asal* \\ *Civil Engineering Department, University of Tabuk, Saudi Arabia \\ fahmy_asal@hotmail.com
}

WG II/4

KEYWORDS: DEM/DTM/DSM, Interpolation, DEM Quality, Visual Analysis, Spatial Analysis

\begin{abstract}
:
Digital elevation data obtained from different Engineering Surveying techniques is utilized in generating Digital Elevation Model (DEM), which is employed in many Engineering and Environmental applications. This data is usually in discrete point format making it necessary to utilize an interpolation approach for the creation of DEM. Quality assessment of the DEM is a vital issue controlling its use in different applications; however this assessment relies heavily on statistical methods with neglecting the visual methods. The research applies visual analysis investigation on DEMs generated using IDW interpolator of varying powers in order to examine their potential in the assessment of the effects of the variation of the IDW power on the quality of the DEMs. Real elevation data has been collected from field using total station instrument in a corrugated terrain. DEMs have been generated from the data at a unified cell size using IDW interpolator with power values ranging from one to ten. Visual analysis has been undertaken using 2D and 3D views of the DEM; in addition, statistical analysis has been performed for assessment of the validity of the visual techniques in doing such analysis. Visual analysis has shown that smoothing of the DEM decreases with the increase in the power value till the power of four; however, increasing the power more than four does not leave noticeable changes on 2D and 3D views of the DEM. The statistical analysis has supported these results where the value of the Standard Deviation (SD) of the DEM has increased with increasing the power. More specifically, changing the power from one to two has produced $36 \%$ of the total increase (the increase in SD due to changing the power from one to ten) in SD and changing to the powers of three and four has given $60 \%$ and $75 \%$ respectively. This refers to decrease in DEM smoothing with the increase in the power of the IDW. The study also has shown that applying visual methods supported by statistical analysis has proven good potential in the DEM quality assessment.
\end{abstract}

\section{INTRODUCTION}

A Digital Elevation Model (DEM) is a continuous surface forms an array of a set of earth's surface points of X (Easting), Y (Northing) and $\mathrm{Z}$ (height). DEM data can be collected or generated using GPS or conventional surveying techniques; analog/analytical/digital Photogrammetry and non imaging airborne techniques including Airborne Laser Scanning (ALS) and Airborne Synthetic Aperture Radar (SAR). The size and location of the project decides on the technique to be used for collecting DEM data. For a project site smaller than 100 acres and covered by tall trees and/or it constitutes urban landscape, the conventional surveying techniques; total station and spirit leveling, are optimal for such a project (Acharya et. al, 2000, Podobnikar, 2009). DEMs are widely used in remote sensing and Geographical Information Systems (GIS) as they are mainly used for orthorectification of images, topographic mapping and engineering design and modeling. Furthermore, DEMs are used in numerous disciplines, ranging from geo-information to Civil Engineering. In various applications DEM serves as inputs for decision making, as examples they are employed in flood hazards analysis (Soleimamani and Modallaldoust, 2008).

Prior to the use of DEMs in various applications it is important to identify their qualities in order to determine the suitability of a certain DEM to the quality standards necessary for a specific application (Acharya and Chaturvedi, 1997). The quality of a
DEM is subjected to some factors such as the density of the sampling points, the spatial distribution of the sampling points, the method of interpolation used, the propagated errors from the source data in addition to other factors (Zhu et. at, 2005, Karl et. al, 2006). Statistical analysis methods has been traditionally employed in the assessment of the DEM quality with neglecting the visual analysis techniques although visual analysis can be very helpful in performing DEM quality assessment that can balance some weaknesses of the statistical methods. Three reasons may answer the question why visual analysis is still underused in the assessment of the DEM quality. First, visual methods are qualitative methods compared to the statistical ones which are considered to be more objective from the point of view of the Geomatics community. Second, the insufficient graphical capabilities of computers until recently can be behind neglecting these methods if it is added to the longer tradition of using statistical methods. Third, visualization of spatial data has traditionally been part of cartography (Podobnikar, 2009). One of the main aims of that paper is to assess the potential of using visual analysis technique in quality assessment of Digital Elevation Models.

Asal and Hassouna, (2007) studied the effect of the interpolation techniques on the quality of the obtained DEM. The outcome of the research indicated that IDW provides better quality DEM than that is given by the Spline method, which smoothes the surface and generates quite noise DEM. The research did not answer the 
question, which value of the power is to be used with the IDW for providing a DEM of specific quality. For that reason it has been suggested that it would be useful to examine IDW of different powers against the quality of the obtained DEM. A test site in a hilly corrugated terrain has been established to the east of Cairo, Egypt. Data has been collected from field using conventional surveying methods where a total station instrument has been used for measuring the three dimensional coordinates $(\mathrm{x}, \mathrm{y}, \mathrm{z})$ of spot points. DEMs have created from the field data using ESRI spatial analysis and 3D analyst working under ArcView GIS 3.2 commercial software package. All parameters have been kept unchanged except the power of the IDW that has been the only parameter allowed to change for the creation of DEMs using powers ranging from one to ten. Quality assessment using visual analysis techniques of DEMs has been undertaken aiming at viewing differences between DEMs generated from IDW with different powers in representing the earth's surface. Also, statistical analysis has been undertaken for the purpose of the assessment of the potential of using visual analysis in the quality assessment of the DEM.

\section{INVERSE DISTANCE WEIGHTING (IDW)}

Elevation interpolation is a complicated operation that can be defined as a process of predicting a value of attribute $\mathrm{z}$ at unsampled site from measurements carried out at neighbouring sites within given neighborhoods. The final result of applying this operation is the production of a continuous surface from spot elevation observations at sparsely located points (Soleimamani and Modallaldoust, 2008). Elevation interpolation could be considered as a spatial filtering process where the input data are not necessarily located at on a continuous grid. Interpolation operations can be expressed in a mathematical command language, however most users will encounter specialist packages so that standard terminology can be used. It may be useful to mention that predicting an elevation value outside the site area from the point data is known as extrapolation (Burrough and McDonnell, 2000). The main purpose of an interpolation operation is the conversion of point data files into continuous fields so that the spatial patterns of these measurements can be compared with the spatial patterns of other entities (Mayers, 1994). Interpolation operation is applied when the discretised surface has different levels of resolution from the required surface. It may also be applied when the continuous surface is represented by different models. Moreover, interpolation operation is performed when the data available does not cover the domain of the area of interest (Lo and Yeung, 2004).

The ideas of proximity adopted by thiessen polygons is combined with the gradual change of the trend surface by the IDW technique. It is assumed that the value of an attribute $\mathrm{z}$ at unvisited point is a distance weighting average of data points occurring within a neighbourhood or window surrounding the unsampled point. The original data points may be located on a regular grid as well as they can be distributed irregularly over an area where interpolation is performed to locations on a denser regular grid in order to produce a map. The weighted moving approach computes the interpolated elevation as (Burrough and McDonnell, 2000, Elumnoh and Shrestha, 2000):

$$
z=\sum_{i=1}^{n} \lambda_{i} z\left(x_{i}\right) \quad \text { and } \sum_{i=1}^{n} \lambda_{i}=1
$$

where the weights $\lambda_{i}$, are given by $\Phi\left(d\left(x, x_{i}\right)\right)$. It is required that the value of $\Phi(d)$ tends to the measured value as $d$ tends to zero. The most common form of $\Phi(d)$ is the inverse distance weighting predictor whose form is (Burrough and McDonnell, 2000, Elumnoh and Shrestha, 2000 ):

$z\left(x_{j}\right)=\sum_{i=1}^{n} z\left(x_{i}\right) d_{i j} r / \sum_{i=1}^{n} d_{i j}^{r}$

where

$x_{j}=$ the points where the surface is to be interpolated,

$x_{i}=$ the data points.

$r=$ the power of the formula, which can be $1,2,3,4, \ldots$.

As in equation (2) $\Phi(d)$ tends to infinity as d tends to zero, then the elevation value of an interpolated point of horizontal coordinates which coincide with those of a data point must be copied over through the process of forcing the inverse distance weighting to the exact values at the data points. In otherwords, if the input grid coordinates are equal to those of a sampling point, then the interpolated elevation at this location will be copied over by the exact elevation value of the sampling point. The quality of the DEM produced from the IDW interpolation may be assessed by using additional observations as the method has no inbuilt technique for testing the quality of the interpolated surface (Burrough and McDonnell, 2000, Lo and Yeung, 2004).

\section{SPOT ELEVATION MEASUREMENTS}

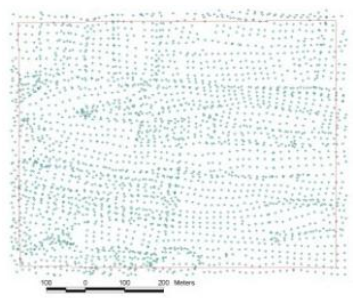

Figure 1: Spot elevation measurements.

Figure 1 shows spot elevation measurements collected from field using a total station instrument in a test site formed of a hilly corrugated terrain and located to the east of Cairo, Egypt. The sample data covers an area of about 830 by 660 metres and consists of 2687 spot elevation measurements forming a density of an elevation point for every $203.87 \mathrm{~m}^{2}$ and an average spacing between spot elevations of about $14.28 \mathrm{~m}$. This area constitutes about $547800 \mathrm{~m}^{2}$, which is very frequently used by the Geomatics Engineers, especially if they use ground Surveying techniques for collecting digital elevation data for medium sized projects. The maximum elevation of data is $138.27 \mathrm{~m}$ and the minimum elevation is $116.73 \mathrm{~m}$ above the mean sea level giving a range of elevations of $21.84 \mathrm{~m}$. The mean elevation is $128.76 \mathrm{~m}$ and the median is $129.62 \mathrm{~m}$ while the mode of elevation records two values of equal frequencies; are $131.96 \mathrm{~m}$ and $131.07 \mathrm{~m}$. Additionally, the variance of the sample is $18.68 \mathrm{~m}^{2}$ and the standard deviation of the mean is $\pm 4.3 \mathrm{~m}$, which is quite high value referring to highly varied terrain. 


\section{GENERATING DIGITAL ELEVATION MODELS USING IDW WITH DIFFERENT POWERS}

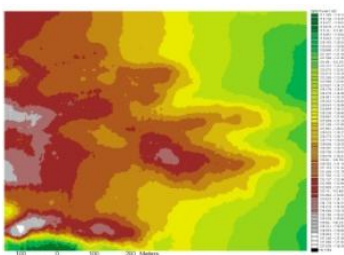

Figure 2: DEM created using IDW of power of one from spot elevation measurements.
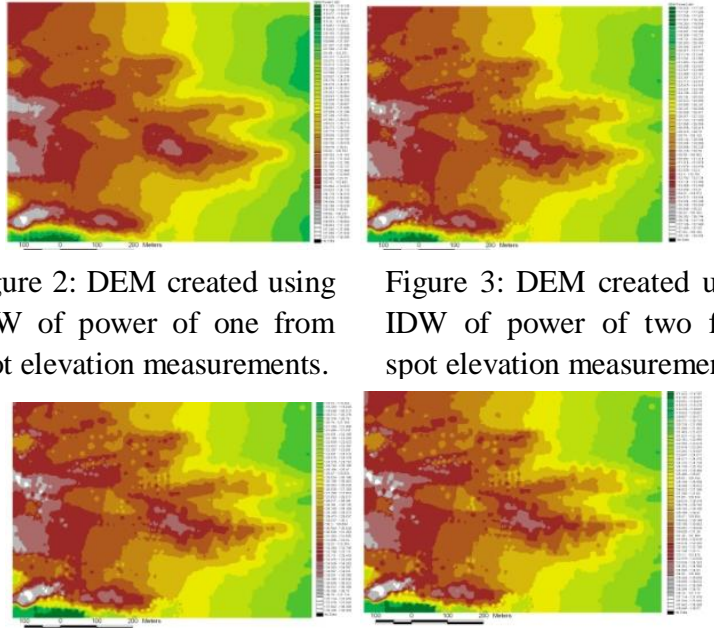

Figure 3: DEM created using IDW of power of two from spot elevation measurements.

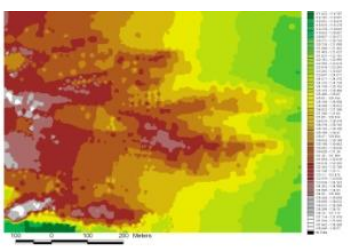

Figure 4: DEM created using Figure 5: DEM created using IDW of power of three from IDW of power of four from spot elevation measurements. spot elevation measurements.

Figures 2 to 11 represent DEMs created from spot elevation data collected from field using a total station instrument. ArcView GIS 3.2 with the spatial analysis and the $3 \mathrm{D}$ analyst extensions have been used in creating the DEMs. The IDW technique has been the algorithm exploited in this process. The grid cell size has been 2.0 metres, in order to minimize the effect of grid sampling that arises as DEM smoothing on the produced DEM, and kept unchanged for all the generated DEMs. The number of neighbours used is 12 as the default input of the system and kept unchanged for all the DEMs as well. The only factor allowed to change is the power of the IDW employed in the creation of the DEM, which is the factor under investigation. Digital Elevation Model is a continuous surface represented by $2 \mathrm{D}$ image in which the attributes of the cells; the elevations are represented by variations in the tone/colour (Burrough and McDonnell, 2000). In this case the elements of digital image interpretation can be exploited in 2D visual interpretation of the DEM. Shape, size, 2D locations of the colour patches in addition to the change in the tone/colour are main criteria that can be applied in 2D visual interpretation of the DEM. Also, the texture which expresses the arrangements and repetition of the tone; smooth, intermediate or rough adding to the pattern which is the arrangements of the spatial objects on the ground are other criteria that can be performed in this analysis (Jensen, 2000, Lillesand and Keifer, 2000).

Referring to figure 2, which depicts a DEM created using IDW with the power of one it is noticed that the tones/colour within different elevation classes are clear while the texture is smooth within the same class with very limited numbers of different tinny colour patches referring to smoothing and approximating of the DEM elevations. The texture is coarser and the view is sharper when changing from one class to another with an unsymmetrical pattern of the elevation classes as the case of the natural terrain. Differently interpretable DEM is depicted in figure 3, which is a DEM generated using the power of two where interactions between different color classes are observable with bigger

numbers of tinny colour patches embedded within bigger different colour patches of different tone representing different colour classes. This refers to bigger changing in the tone and coarser texture with random elevation patterns leading to better interpolation of the elevations in a highly varied terrain and less smoothing of the DEM. Additionally, less sharpness between colour classes is observable in figure 4 , which is a DEM created using IDW of power of three with larger numbers of the different tone-tinny colour patches and more coarser texture with random pattern reflecting more corrugations in the DEM and consequently less smoothing of the terrain. Smaller differences between DEMs may be interpretable in figures starting from figure 5, which is a DEM created with the power of four to figure 11, which is a DEM created with the power of ten. This reflects smaller improvements in the DEM characteristics with the increase in the value of the power of the IDW more than four.

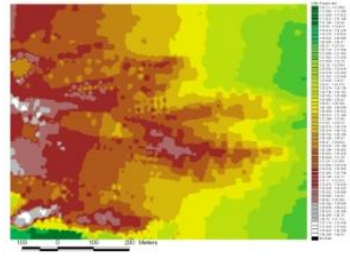

Figure 6: DEM created using IDW of power of five from spot elevation measurements.

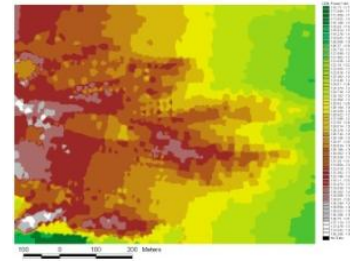

Figure 8: DEM created using IDW of power of seven from spot elevation measurements.

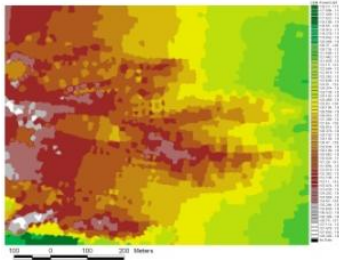

Figure 10: DEM created using IDW of power of nine from spot elevation measurements.

\section{3D VISUALIZATION OF DIGITAL ELEVATION MODELS}

Figures from 12 to 21 are $3 \mathrm{D}$ views generated from digital elevation models created using IDW of different powers, where Figure 12 is a 3D view of DEM generated from IDW of power of one and figure 13 is a $3 \mathrm{D}$ view of DEM interpolated from IDW of power of two. In addition, figures 14 and 15 are 3D views of DEMs created using IDW of powers of three and four respectively. Moreover, figures 16 and 17 are 3D views of DEMs 
interpolated using IDW of powers of five and six respectively. Furthermore, figures 18 and 19 are 3D views obtained from DEMs interpolated using IDW of powers of seven and eight respectively. Finally, figures 20 and 21 are 3D views of DEMs produced using IDW of powers of nine and ten respectively. In addition to the $2 \mathrm{D}$ visual interpretation criteria described section 4 , the height/depth of objects and shadow of objects are main criteria for 3D interpretation of DEM (Jensen, 2000, Lillesand and Keifer, 2000).

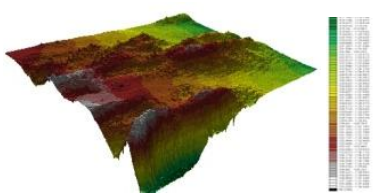

Figure 12: 3D view of the DEM created using IDW of power of one from spot elevation measurements.

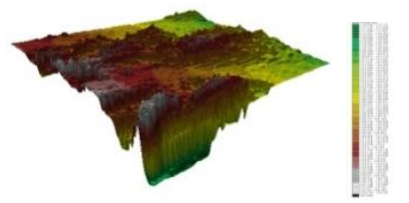

Figure 14: 3D view of the DEM created using IDW of power of three from spot elevation measurements.

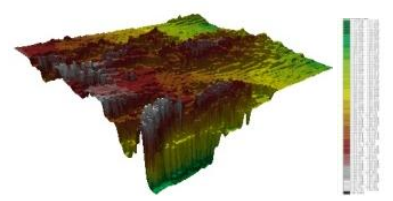

Figure 16: 3D view of the DEM created using IDW of power of five from spot elevation measurements.

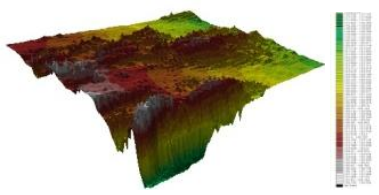

Figure 13: 3D view of the DEM created using IDW of power of two from spot elevation measurements.

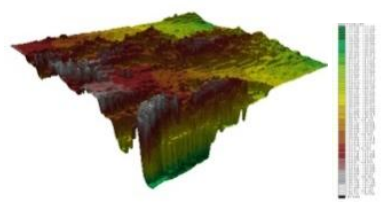

Figure 15: 3D view of the DEM created using IDW of power of four from spot elevation measurements.

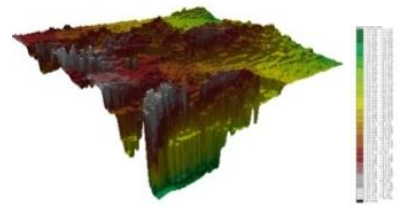

Figure 17: 3D view of the DEM created using IDW of power of six from spot elevation measurements.
$3 \mathrm{D}$ views in Figures 12 to 21 record differences between DEMs generated using IDW of different powers. Compared to the other $3 \mathrm{D}$ views, figure 12 records minimum corrugations that are interpretable from the relatively smaller heights and smaller sizes of the corrugations in the represented terrain. This coincides with the outcomes from the analysis in the $2 \mathrm{D}$ views and interpreted as smoothing of the surface and approximating the DEM elevations. More corrugated surface with the increase in the corrugation of bigger sizes and heights are viewed in figure 13, which has been created using the power of two, leading to less smoothing of the texture, more random pattern as the case of the natural terrain and consequently better estimation of the surface elevations. With increasing the power of the IDW, more corrugated surface might be interpretable from the coarser texture and random pattern of corrugation. This in turn is an indication of improvements in the estimation of the DEM elevations; however, those improvements are much clearer when increasing the power from one to two and become noticeable when increasing the power from two to three and from three to four. Corrugations of the surface increase with increasing the value of power from four to five and so on, but the little changes in the view of the DEM are interpretable with the use of higher values of the IDW power. This analysis shows that $3 \mathrm{D}$ visualization of the DEM can be a useful tool for viewing differences occurring in the DEM due to changing the IDW power.

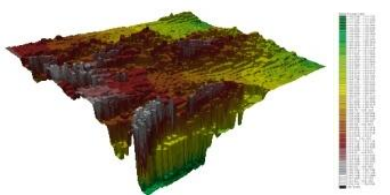

Figure 18: 3D view of the DEM created using IDW of power of seven from spot elevation measurements.

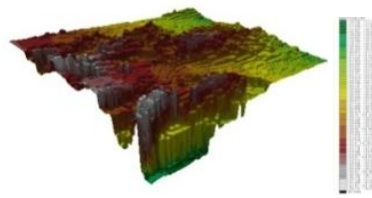

Figure 20: 3D view of the DEM created using IDW of power of nine from spot elevation measurements.

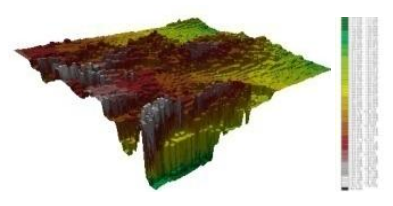

Figure 19: 3D view of the DEM created using IDW of power of eight from spot elevation measurements.

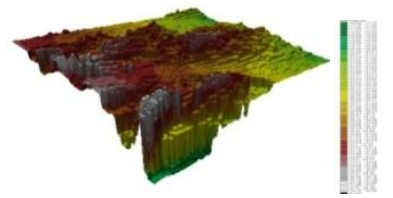

Figure 21: 3D view of the DEM created using IDW of power of ten from spot elevation measurements.

\section{STATISTICAL ANALYSIS OF DEMS CREATED USING DIFFERENT POWERS}

Referring to values in table 1 and the graph in figure 22 it is seen that the sum of elevations in the DEM decreases with the increase in the power of the IDW model from one to ten. However, the decrease in the sum of elevations is rapid and sharp until the use of power of four and becomes milder till the use of power seven while it is very small when using powers more than seven until ten. As a percentage the decrease in the sum of elevations due to changing the power from one to two is about $40 \%$ of the total decrease in the sum of elevations that occurs due to changing from the power of one to the power of ten. When using a power of three the percentage of decrease is about $66 \%$ and becomes about $80 \%$ when using a power of four. Moreover, the percentage of decrease becomes $88 \%$ when using a power of five and $93 \%$ when using a power of six. Additionally, using the power of seven causes a decrease of $96 \%$. Furthermore, increasing the power of the IDW from seven to ten causes slight changes in the sum of elevations in the DEM.

From table 1, when examining the maximum elevations in the DEMs, it is noticed that IDW with power of one records slight decrease in the maximum value compared to that of the original data, which increases with the increase in the power value and approaches the value recorded in the original data with the use of a power of three. The opposite can be said for the minimum elevation where IDW of power of one gives higher minimum elevation value than that is recorded in the original data. This 
value decreases with the increase in the power value approaching the recorded value in the original data with the use of power value of four and remains unchanged due to increasing the power value more than four. The effects of changes in the maximum and minimum elevations are reflected on the range of elevations in the DEM given in table 2. IDW with power of one records narrower range of elevations, 20.48 metres, compared to that recorded in the original data, $21.84 \mathrm{~m}$. The range of elevations in the DEM increases with the increase in the power of the IDW until reaching the value of $21.84 \mathrm{~m}$ with the use of power of four and remains unchanged after that.

\begin{tabular}{|c|c|c|c|c|}
\hline $\begin{array}{c}\text { Statistical } \\
\text { Value }\end{array}$ & $\begin{array}{c}\text { Sum } \\
\text { (metres) }\end{array}$ & $\begin{array}{c}\text { Max. } \\
\text { (metres) }\end{array}$ & $\begin{array}{c}\text { Min. } \\
\text { (metres) }\end{array}$ & $\begin{array}{c}\text { Mean } \\
\text { (metres) }\end{array}$ \\
\hline DEM-P1 & 17458377 & 138.270 & 117.790 & 128.567 \\
\hline DEM-P2 & 17454423 & 138.550 & 116.840 & 128.538 \\
\hline DEM-P3 & 17451839 & 138.570 & 116.740 & 128.519 \\
\hline DEM-P4 & 17450394 & 138.570 & 116.730 & 128.508 \\
\hline DEM-P5 & 17449597 & 138.570 & 116.730 & 128.502 \\
\hline DEM-P6 & 17449133 & 138.570 & 116.730 & 128.499 \\
\hline DEM-P7 & 17448849 & 138.570 & 116.730 & 128.497 \\
\hline DEM-P8 & 17448666 & 138.570 & 116.730 & 128.496 \\
\hline DEM-P9 & 17448540 & 138.570 & 116.730 & 128.495 \\
\hline $\begin{array}{c}\text { DEM- } \\
\text { P10 }\end{array}$ & 17448452 & 138.570 & 116.730 & 128.494 \\
\hline
\end{tabular}

Table 1: The statistical properties elevations in the DEMs created using IDW interpolator with different powers.

\begin{tabular}{|c|c|c|c|c|}
\hline $\begin{array}{c}\text { Statistical } \\
\text { Value }\end{array}$ & $\begin{array}{c}\text { Median } \\
\text { (metres) }\end{array}$ & $\begin{array}{c}\text { Mode } \\
\text { (metres) }\end{array}$ & $\begin{array}{c}\text { Range } \\
\text { (metres) }\end{array}$ & $\begin{array}{c}\text { Standard } \\
\text { Deviation } \\
\text { (metres) }\end{array}$ \\
\hline DEM-P1 & 129.400 & 131.370 & 20.480 & 3.926 \\
\hline DEM-P2 & 129.320 & 131.350 & 21.710 & 3.969 \\
\hline DEM-P3 & 129.260 & 131.090 & 21.830 & 3.998 \\
\hline DEM-P4 & 129.230 & 131.070 & 21.840 & 4.015 \\
\hline DEM-P5 & 129.200 & 131.070 & 21.840 & 4.026 \\
\hline DEM-P6 & 129.180 & 131.070 & 21.840 & 4.032 \\
\hline DEM-P7 & 129.170 & 131.070 & 21.840 & 4.037 \\
\hline DEM-P8 & 129.170 & 131.070 & 21.840 & 4.040 \\
\hline DEM-P9 & 129.160 & 131.070 & 21.840 & 4.043 \\
\hline $\begin{array}{c}\text { DEM- } \\
\text { P10 }\end{array}$ & 129.160 & 131.070 & 21.840 & 4.045 \\
\hline
\end{tabular}

Table 2: The statistical properties elevations in the DEMs created using IDW interpolator with different powers.

This means that smoothing of elevations is at its highest with power of one and decreases with the increase in the power of the IDW. Also, when examining the Standard Deviation (SD), with referring to table 2 and figure 23, which depict the effects on SD of the elevations in DEMs due to changes in the IDW power, it is observed that SD increases with the increase in the power of the IDW technique. The increase in SD is sharp till reaching the power of four where about $75 \%$ of the total increase is obtained and become smaller with powers of five, six and seven where about $93 \%$ of the total increase is recorded at power of seven. It is important to note here that the increases in SD of elevations in the DEM refer to increases of variations of elevations in the DEM, which is a reflection of decreasing in smoothing of DEMs and consequently better estimation of elevations.

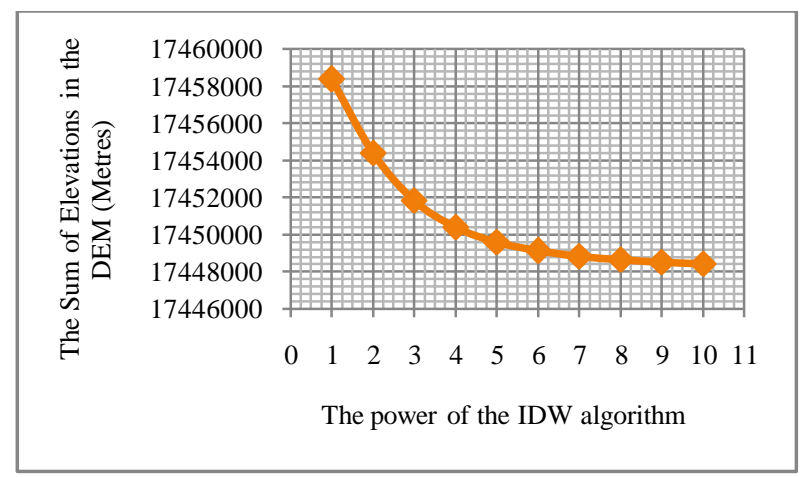

Figure 22: The effect of changing the power of the IDW on the sum of the elevations in the generated DEM.

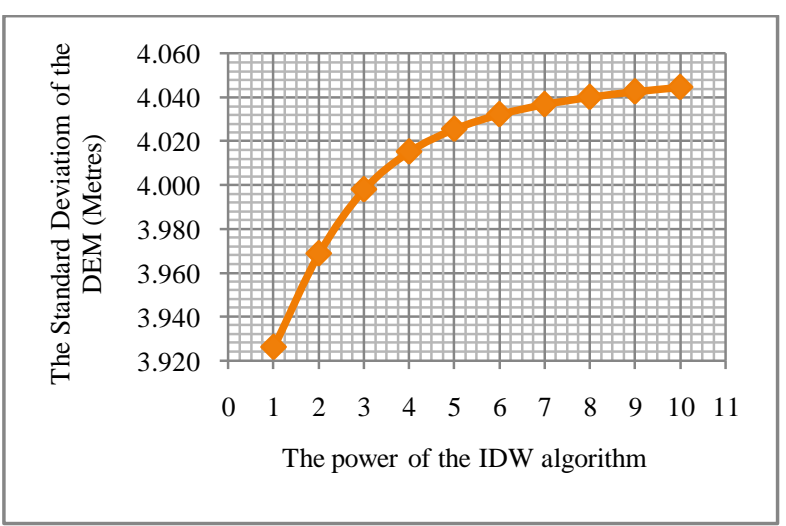

Figure 23: The effect of changing the power of the IDW on the standard deviation of the elevations in the generated DEM.

\section{DISCUSSIONS}

DEMs have been created from a point support file containing 2687 discrete spot elevations using IDW interpolation algorithm with different powers starting from power of one to power of ten giving ten different DEMs. All the factors of the IDW algorithm have been kept unchanged except the power factor, the factor under examination. Three tests have been performed on DEMs in order to assess changes in the DEM quality due to changes in IDW power. Visual analysis of 2D views has been performed, where elements of image interpretation including the size, shape, tone/colour, pattern, and height and depth have been employed in such analysis. DEM created using the power of one has shown clear view with sharp transition between elevation classes and less changes in the tone within the DEM and relatively smooth texture which reflects elevation smoothing and less quality DEM. DEM produced using the power of two is of highly varied tone, of less, clearer, less bright and less sharper view. It is also, of coarser texture with more random pattern than that one created using the power of one leading to more reliable elevation interpolation. More improvements in the DEMs have been due to change to power of three and also, to power of four, however, DEMs created using powers of five to ten are similar in views where it is difficult for differences to be distinguishable. Visual analysis of the $3 \mathrm{D}$ views has shown texture smoothing and less corrugated surface in figure 12 , which is a $3 \mathrm{D}$ view of the DEM created using the power of one. Interpolation of elevations has improved when using the power of two where a more corrugated surface viewed 
in bigger heights and sizes of corrugations in the $3 \mathrm{D}$ view. With the use of power of three the corrugations increase, but with smaller rates. Additionally, when increasing the power more than four, the changes in the surface become hardly noticeable, which coincides with the outcomes from the analysis of the $2 \mathrm{D}$ views.

Statistical analysis of the DEMs has been carried out so that some of the statistical properties of the generated DEMs can be examined against the use of different powers. Clear differences in the statistical properties of DEMs have been obtained where the sum of elevations in the DEM decreases with the increase in the value of the power. Considering the total decrease in the sum of DEM elevations due to changes in the power of the IDW from one to ten is one hundred percent, increasing the power from one to two has produced about $40 \%$ of the total decrease. Additionally, the use of power of three has produced about $66 \%$ of the total decrease (i.e. about two thirds of the total decrease). Furthermore, the use of power of four has caused a decrease of about $80 \%$ of the total decreases in the values of the sum of elevations in the DEM. In addition to that, the values of the maximum, the minimum, the range, the mode and the median elevations record some changes from their corresponding values in the original data when using powers of; one, two, three and four, however, with the use of powers higher than four the corresponding values of the maximum, minimum, range, mode and median elevations in the original data has been retained. These results are supported by the Standard Deviation (SD) of the DEM, where, changing the power from one to two produces an increase in the SD of about $36 \%$ of the total increase. However, with the use of power of three this percentage becomes about $60 \%$ where it increases to $75 \%$ when using the power of four. On the other hand, increasing the power of the IDW more than four has given increases in the values of the standard deviation, but the rate of increasing becomes slighter.

\section{CONCLUSIONS}

Quality assessment of the DEM constitutes a vital issue that controls its employment in a wide range of applications. As a tradition quality assessment used to be performed using statistical techniques with neglecting the visual methods (Podobnikar, 2009). The research aimed at assessment of the potential of visual analysis methods in viewing differences in DEMs generated from IDW of varying powers with the indication of how the obtained results of the visual analysis conform with those from the statistical analysis. The study has indicated that IDW with the power of one gives the highest degree of texture smoothing and approximation of DEM elevations. Also, visual analysis has shown increase in DEM corrugations interpreted from the coarser textures, random patterns, increasing of size and height of the corrugations due to the increase in the power value more than one. On the other hand, increasing the power more than four has produced little changes in the DEM characteristics till the power of seven while those changes in the employed visual interpretation criteria including shape, size and height of corrugations, variations in the tones/colours in the DEM, changes in the textures and patterns of the DEM are hardly noticeable with higher powers. Such results have been supported by the outcomes from the statistical analysis where increasing the power of the IDW from one to two has provided about $36 \%$ of the total increase in SD (the increase due to changing the power from one to ten). Additionally, increasing the power from one to three has given about $60 \%$ increase in the SD, while with the increase from one to four an increase of about $75 \%$ has been the result. Visual analysis has shown great potential in quality assessment, especially if they are supported by statistical analysis techniques. Deeper and thorough investigations could be necessary in order to evaluate how it is worthy of using IDW of powers more than four which gives little improvements of the DEM quality against the other factors that could be increasing in the computational time and higher specifications of the computers required for such processing.

\section{ACKNOWLEDGMENTS}

The author would like to acknowledge the three anonymous reviewers who helped strengthening the paper.

\section{REFERENCES}

Acharya, B. and Chaturvedi, A., 1997. Digital Terrain Model: Elevation Extraction and Accuracy Assessments. Journal of Surveying Engineering, Vol.123, No.2, May 1997, pp. 71-76.

Acharya, B., Fagerman, J. and Wright, C., 2000, Accuracy Assessment of DTM Data: a Cost Effective Approach or a Large Scale Digital Mapping Project. International Archives of Photogrammetry, Remote Sensing and Spatial Information Sciences, Amsterdam, The Netherlands, Vol. XXXIII.

Asal, F. F. and Hassouna, R., 2007. Accuracy Assessments of Digital Elevation Models Created Using Different Interpolation Techniques. Al-Azhar Univ. Eng. Journal, JAUES Vol. 2, No. 3. Burrough, P. A. and McDonnell, R. A. 2000. Principles of Geographical Information Systems. Oxford University Press.

Elumnoh, A. and Shrestha,R. P., 2000. Application of DEM Data to Landsat Image Classification: Evaluation in a Tropical WetDry Landscape of Thailand. Photogrammetric Engineering \& Remote Sensing. Vol. 66, No. 3, March 2000, pp. 297-304.

Jensen, J., 2000. Remote Sensing of the Environment: An Earth Resource Perspective, Prentice Hall, New Jersey 07458.

Karl, W., Pfeifer, N. and Briese, C., 2006. DTM Quality Assessment. Working Group 11/7, 2006.

Lillesand, T. M. and Keifer, R. W., 2000. Remote Sensing and Image Interpretation. Fourth Edition, John Wiley \& Sons, Inc.

Lo, C. P. and Yeung, A. K. W., 2004. Concepts and Techniques of Geographic Information Systems. Prentice-Hall of India Private Limited, New Delhi - 110 001, 2004.

Mayers, E. D., 1994. Spatial Interpolation: An overview. Geoderma, 62: 17-28, 1994.

Podobnikar, T., 2009. Methods for Visual Quality Assessment of Digital Terrain Model. Journal of SAPIENS (Surveys and Perspectives Integrating Environment and Society), Vol. $2 / \mathrm{n}^{\circ} 2$ Special issue. http://sapiens.revues.org/index738.html.

Soleimamani, K. and Modallaldoust, S. 2008. Production of Optimized DEM Using IDW Interpolation Method (Case Study; Jam and Riz Basin-Assaloyeh). Journal of applied Sciences 8(1): 104-111, 2008, ISSN 1812-5654,

Zhu, C., Shi, W., Li, Q., Wang, G., Cheung, T. C. K., Dai, E., and Shea, G. Y. K., 2005. Estimation of Average DEM Accuracy under Linear Interpolation Considering Random Errors at the Nodes of TIN Model. International Journal of Remote Sensing, Volume 26, Number 24,01D2005, pp. 5509-5523(15). 